\title{
Detection of neurological damage during open-heart surgery
}

\author{
M. A. B R A N T HW A I T E \\ Brompton Hospital, London
}

\begin{abstract}
Branthwaite, M. A. (1973). Thorax, 28, 464-472. Detection of neurological damage during openheart surgery. Cerebral activity during open-heart surgery has been recorded in 140 patients using a heavily filtered electroencephalograph, the Cerebral Function Monitor (CFM). Unlike the conventional electroencephalogram the CFM record is recorded at slow speeds, is filtered to minimize electrical interference, and is easy to interpret.

A high incidence $(62.9 \%)$ of alteration in electrical activity was noted at the onset of perfusion, and several different patterns of change are described.

Ten patients suffered neurological damage to a degree which produced physical signs in the postoperative period. In eight of these 10 cases, abnormal depression of the CFM record was noted during perfusion; the depression occurred at the onset on five occasions. Equivocal changes were noted at the onset of perfusion in one patient whose neurological lesion may have been related to a postoperative cardiac rhythm disorder. One patient sustained a midbrain lesion, probably at the onset of perfusion, and the CFM record during operation was unremarkable.

The incidence of both neurological damage and depression of the CFM record at the onset of perfusion has decreased since filters for microemboli have been included in the perfusion circuit.

It is concluded that the CFM is a useful means of detecting acute changes in cortical activity during open-heart surgery. The risk of cerebral damage is high at the onset of perfusion when both hypotension and microemboli from the extracorporeal circuit may contribute to the neurological lesion.
\end{abstract}

A previous study of neurological damage during open-heart surgery revealed an incidence of approximately $20 \%$ (Branthwaite, 1972). Three factors appeared to be associated, namely age, duration of perfusion, and the presence of preexisting neurological disease. It proved impossible to identify precise causes with any certainty.

Recording the electro-encephalogram (EEG) throughout the procedure has been advocated as a means of monitoring cerebral function (FischerWilliams and Cooper, 1964), and previous studies have noted events which are commonly associated with EEG changes, such as cannulation or obstruction of the superior vena cava (Pampiglione and Waterston, 1961) and acidosis (Brazier, 1961). Conventionally, the EEG is recorded from multiple sites at fast speed. Interference due to artefacts is common (Sadove, Becka, and Gibbs, 1967), and considerable skill and experience are required for accurate interpretation. Recently, a cerebral function monitor
(CFM) has been developed which records a $\frac{0}{3}$ heavily filtered EEG signal at slow speeds (Maynard, Prior, and Scott, 1969). Acute changes in cerebral activity cause a change in either the mean value or amplitude of the tracing and are 5 easily recognized by the observer; interference is $\frac{D}{0}$ eliminated to a large extent by the filter system.

Relative freedom from interference together $N$ with the ease of interpretation and slow speed of $N$ recording form a combination which is particu- $N$ larly suitable for monitoring cardiac surgery, and $\mathrm{\omega}$ Prior et al. (1971) have described its use for this purpose in 49 cases. In the present study, an

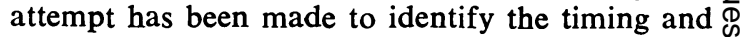
hence the presumptive nature of neurological damage by recording the EEG throughout the operation using the CFM.

\section{MATERIAL AND METHODS}

One hundred and forty patients have been studied during open-heart surgery for a wide variety of con- 
genital and acquired heart disease. Their ages ranged from 3 months to 74 years.

In the majority of cases anaesthesia consisted of premedication with omnopon and scopolamine followed by a thiopentone, nitrous oxide-oxygen-relaxant sequence, supplemented when necessary with either omnopon or low concentrations (less than $1 \%$ ) of halothane.

There was considerable variation in perfusion technique and apparatus; four types of oxygenator were used (rotating disc, Rygg, Temptrol, and membrane) and different degrees of haemodilution were employed. The $\mathbf{p H}$ of the perfusate was adjusted to approximately 7.4 with sodium bicarbonate before the onset of perfusion. In the first 33 cases the arterial return was filtered through a $150 \mu$ mesh and bubble trap, but in later cases a Swank filter for microemboli was incorporated into the cardiotomy suction and blood priming line; more recently, a $40 \mu$ Barrier filter ${ }^{1}$ has been used in addition on the arterial return.

Perfusion was carried out at a normal or only moderately reduced temperature $\left(30-35^{\circ} \mathrm{C}\right)$ in all but seven cases, in five of whom hypothermic circula-

1Johnson and Johnson tory arrest was employed at a nasopharyngeal temperature between 15 and $27^{\circ} \mathrm{C}$. In the majority of patients the arterial carbon dioxide tension was maintained between 30 and $40 \mathrm{mmHg}$ throughout the operation. Routine monitoring included arterial and superior vena caval pressures, nasopharyngeal temperature, and arterial blood gas tensions and $\mathrm{pH}$.

The EEG was recorded from two intradermal platinum needle electrodes ${ }^{2}$ situated in the parietal region on either side of the midline, approximately $5 \mathrm{~cm}$ apart and a little behind the level of a line joining the external auditory meati. The impedance between the two electrodes was monitored continuously and was always well below $5 \mathrm{~K}$. The CFM was a prototype instrument, the definitive version of which is now available from Devices Ltd.

At first both the impedance and CFM signals were recorded on a Devices 2-channel pen recorder running at $6 \mathrm{~mm} / \mathrm{min}$; subsequently, an SE Laboratories multi-channel ultraviolet recorder was used at a speed of $10 \mathrm{~mm} / \mathrm{min}$. This enabled additional information to be recorded simultaneously, in particular, the arterial and superior vena caval pressures and the nasopharyngeal temperature.

2Specialized Laboratory Equipment
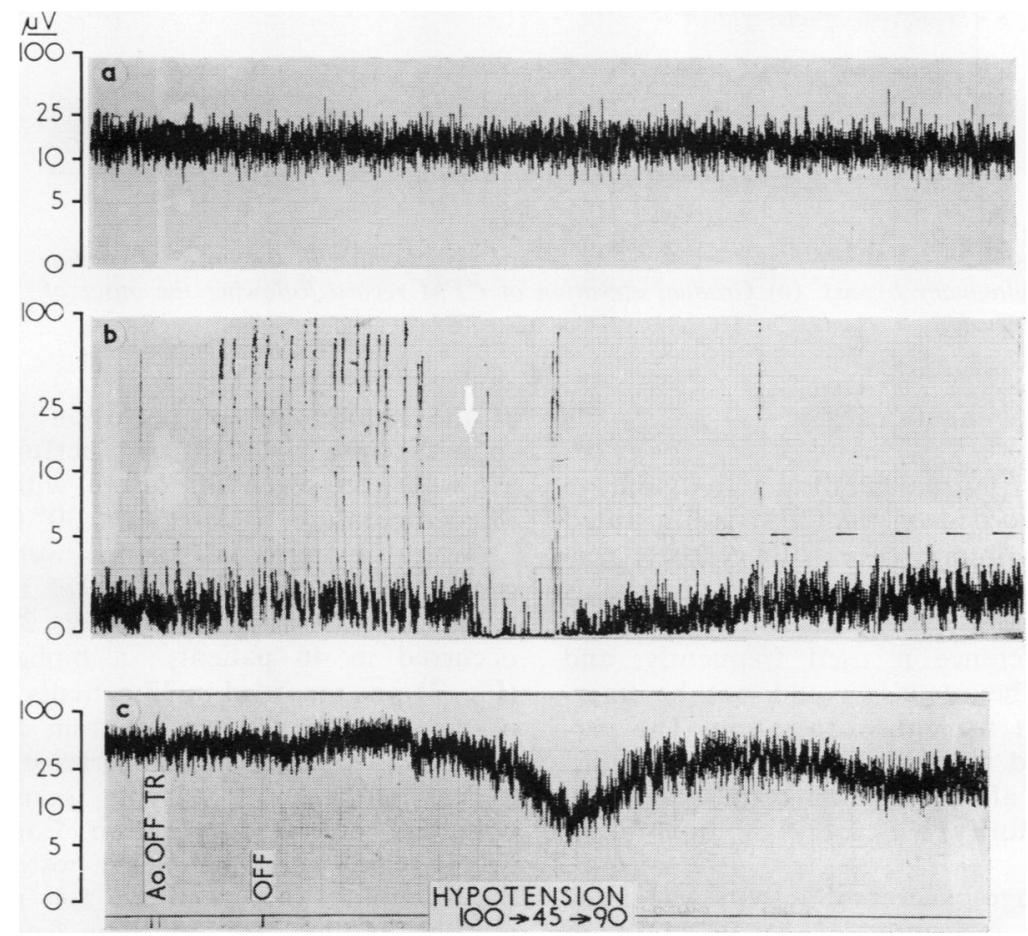

FIG. 1. (a) Normal CFM record obtained during anaesthesia. (b). Virtual abolition of cerebral activity during an episode of ventricular fibrillation (onset at arrow). The high voltage activity preceding this represents interference due to surgical diathermy. (c) Depression of cerebral activity associated with hypotension due to haemorrhage and administration of protamine. 


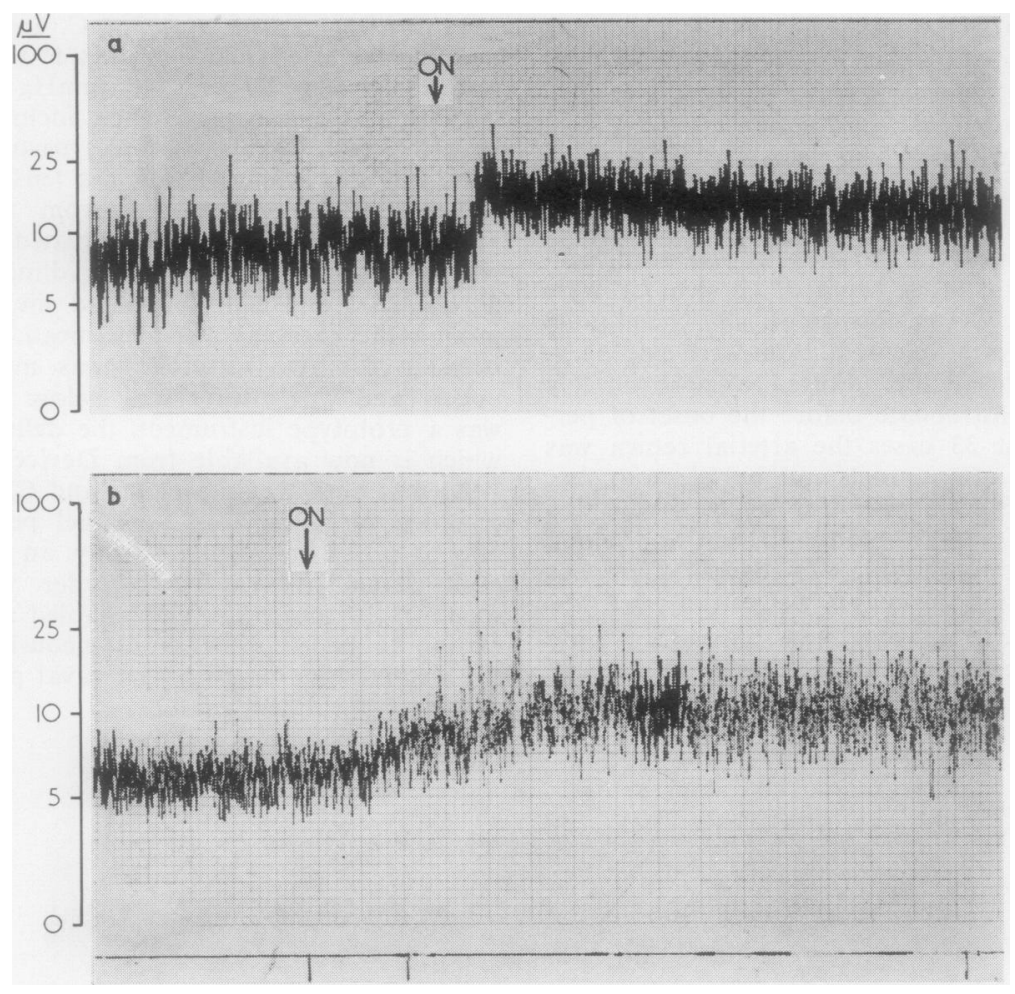

FIG. 2 (a) Acute elevation of CFM record associated with the onset of cardiopulmonary bypass. (b) Gradual elevation of CFM record following the onset of perfusion.

\section{RESULTS}

The appearance of normal cerebral activity during anaesthesia as recorded on the CFM is illustrated (Fig. 1a). Sudden interference with cerebral perfusion produces an abrupt change in the record as shown in Fig. 1b and c. Surgical diathermy causes prohibitive interference if used frequently, and for this reason the records could not be interpreted throughout the entire operation. The perfusion period and an interval of 15 minutes or more before and after perfusion could always be recorded satisfactorily with only minimal interference (Fig. 1b).

An abrupt change in cerebral activity was noted on the CFM at the onset of perfusion in 88 patients $(62 \cdot 9 \%)$. In two patients there was a slight increase in the mean level of the record when cannulation of the superior vena cava was associated with an increase in caval pressure, and in 40 patients there was a slight drop in mean level at the termination of perfusion. In 16 patients $\frac{3}{3}$. changes were noted during perfusion and were $\delta$ associated in seven of the 16 with reduction of $₹$ the temperature to less than $30^{\circ} \mathrm{C}$.

Several patterns of change were seen at the onset of perfusion. Elevation of the record was either sudden or gradual (Fig. 2a and b) and $\bar{N}$ occurred in 46 patients; a biphasic movement 0 (Fig. 3) was recorded in 22 patients and depression $N$ of activity (Fig. 4) was seen in 20 patients.

Thirteen patients in this series sustained neuro- $\bar{O}$ logical damage of varying severity, apparent 0

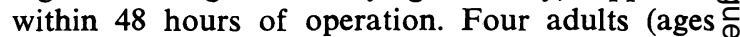
53-60 years) were hemiplegic postoperatively and $\stackrel{\mathcal{D}}{+}$ the disability has persisted for more than six 0 months in one case. In these four patients, the 0 only abrupt change in the CFM record was at $\stackrel{\mathbb{D}}{\stackrel{D}{Q}}$ the onset of perfusion when there was a marked $\mathbb{Q}$ and sustained depression with slow, high-voltage $\frac{\sigma}{\sigma}$ activity persisting for some minutes. Figure 5 is a representative example and also illustrates the 


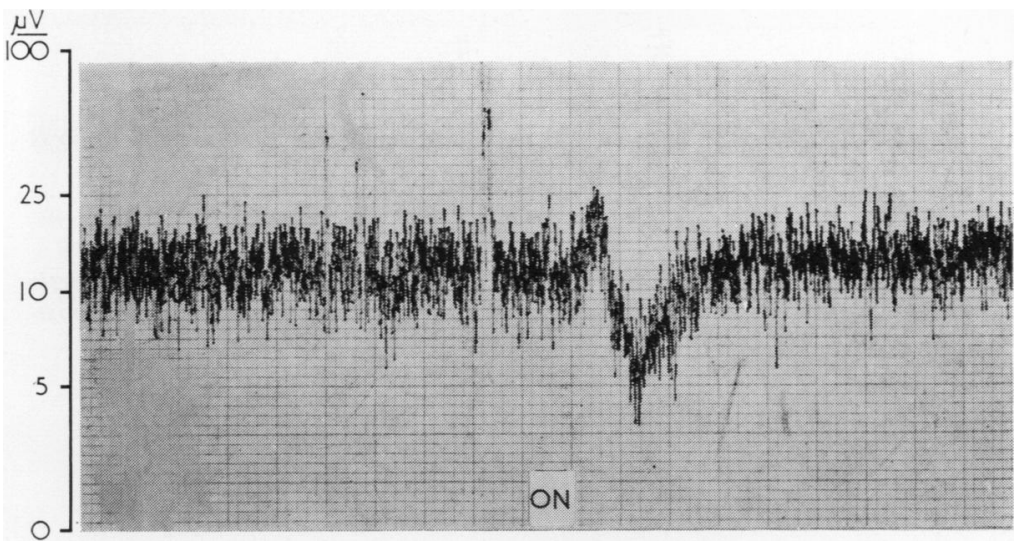

FIG. 3. Biphasic change at the onset of perfusion.

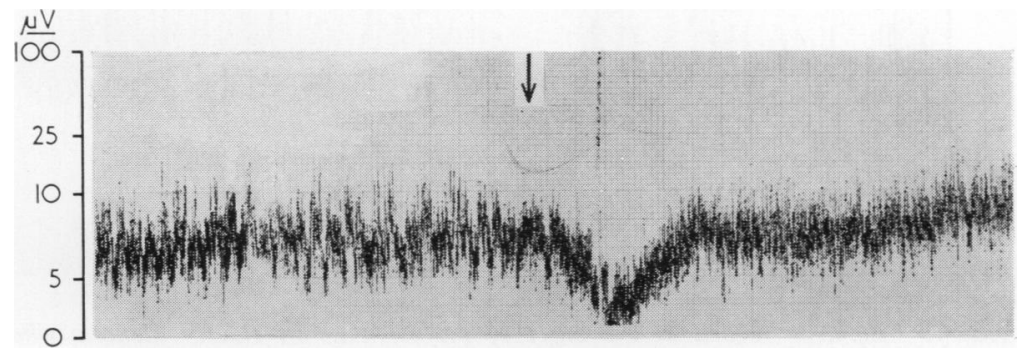

FIG. 4. Depression of CFM record following the onset of perfusion.

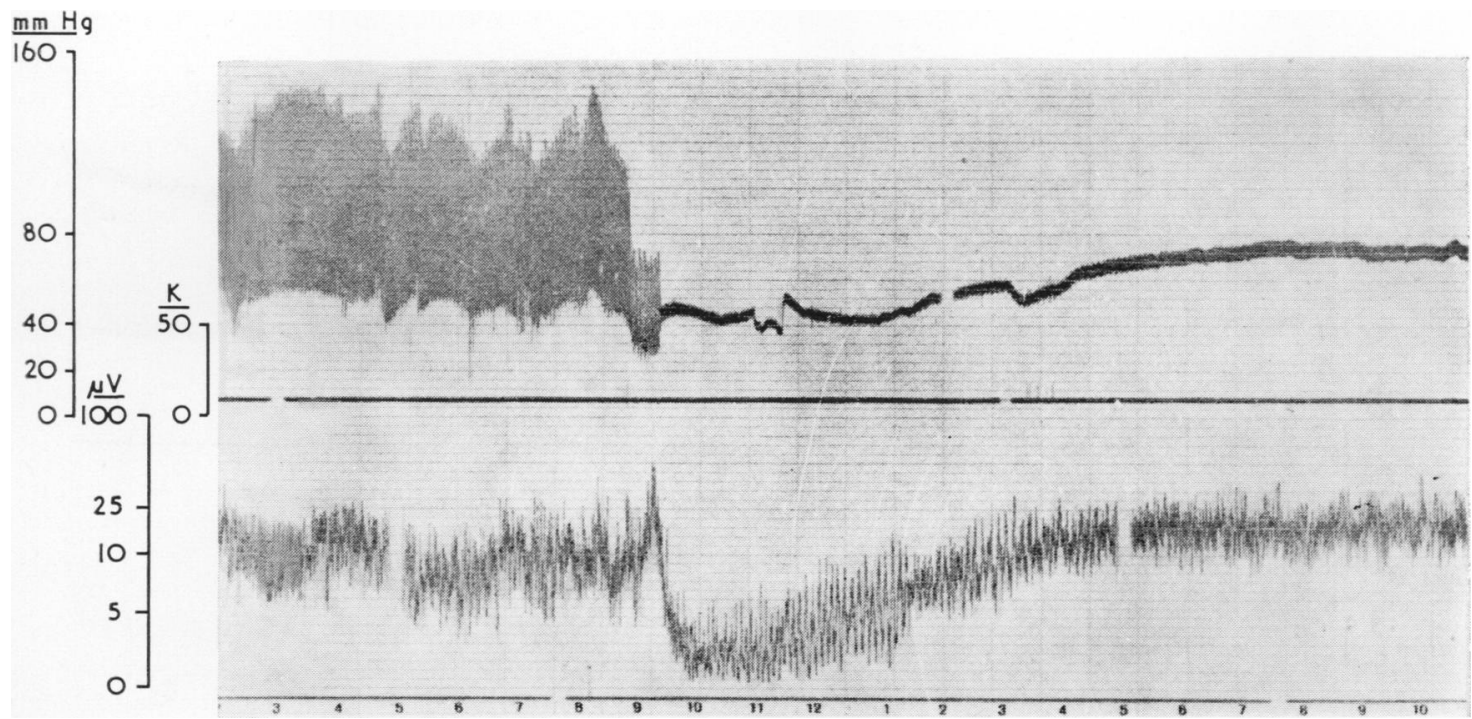

FIG. 5 Profound depression of CFM record at the onset of perfusion. The drop is followed by a period of high-voltage, slow activity, taking 10 minutes to recover. Tracings from above downwards are arterial blood pressure, impedance between the two CFM electrodes, and the CFM record itself. This patient was hemiplegic postoperatively. 


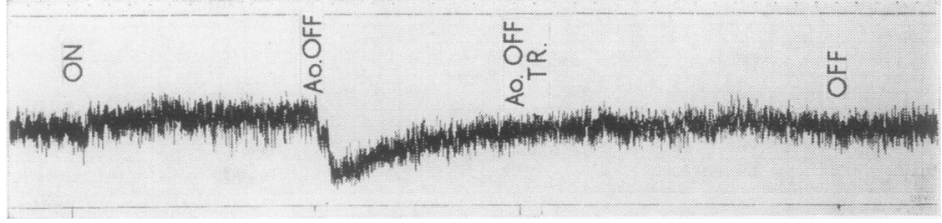

FIG. 6. Acute depression of CFM record following release of the aortic clamp during perfusion. This patient had a transitory monoplegia postoperatively.

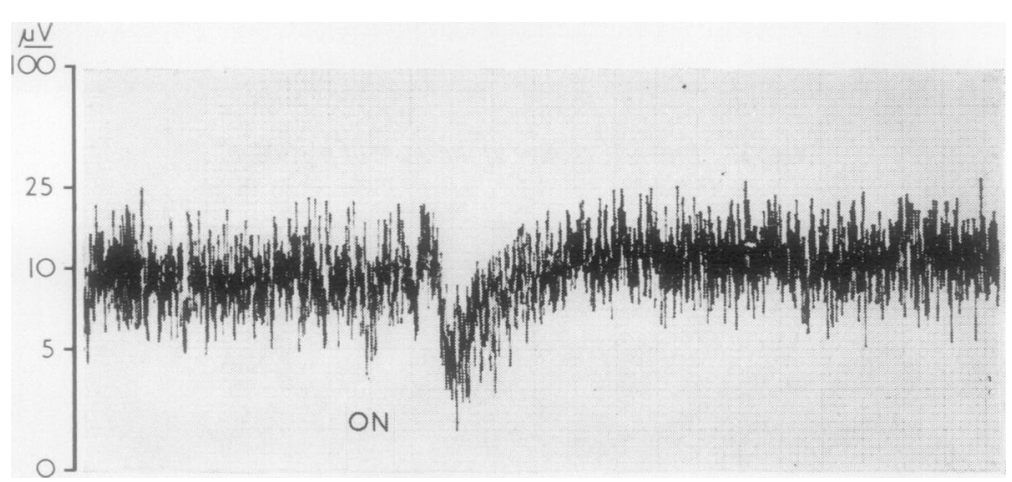

FIG. 7. Biphasic record at the onset of perfusion in an elderly diabetic who suffered an epileptic fit on the second postoperative day.

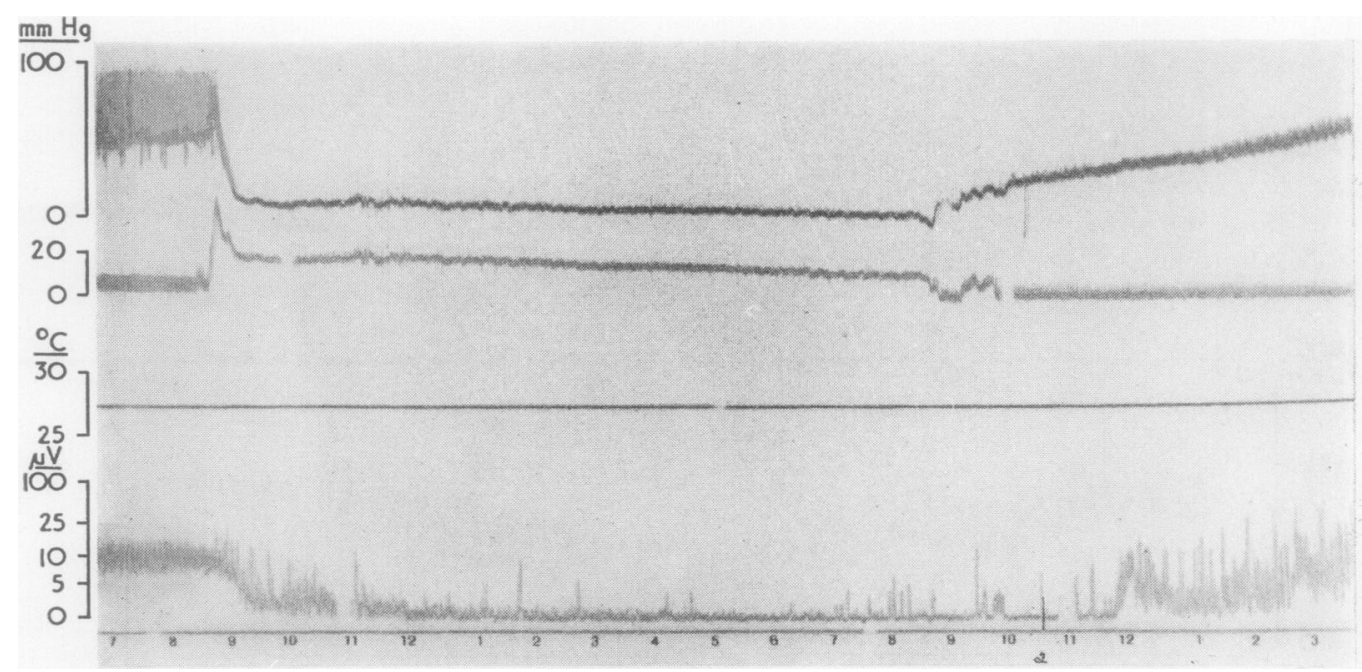

FIG. 8. Depression of CFM record associated with circulatory arrest at a nasopharyngeal temperature of $27^{\circ} \mathrm{C}$ in a 1-year-old child. Records from above down are arterial blood pressure, superior vena caval pressure, nasopharyngeal temperature, and the CFM record. Duration of circulatory arrest was $14 \frac{1}{2}$ minutes. 


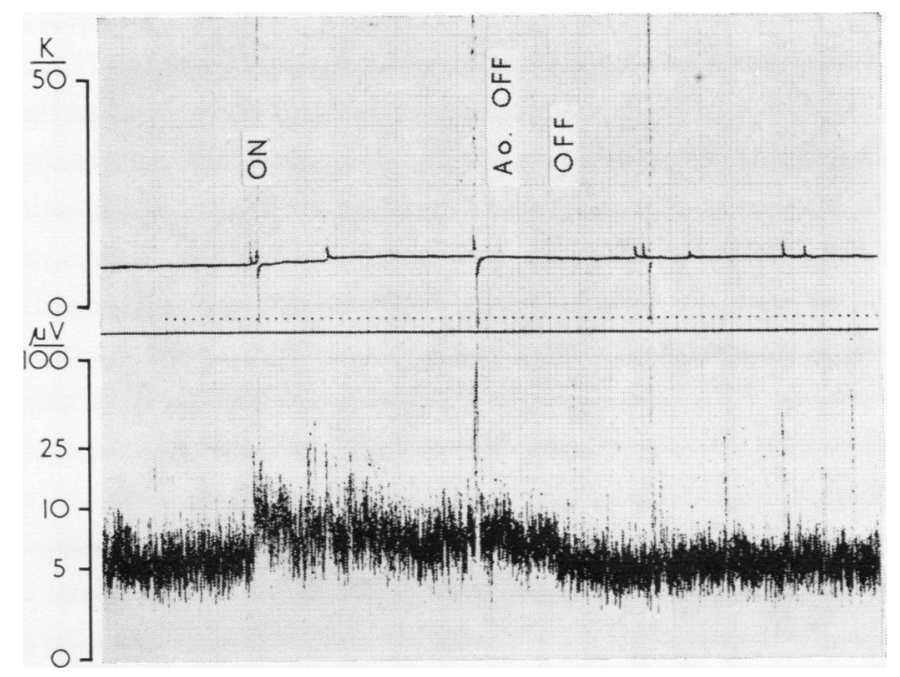

FIG. 9. Complete perfusion record at very slow speed $(1.5 \mathrm{~mm} / \mathrm{min})$ in a patient who sustained a mid-brain lesion during surgery. Total perfusion time: 35 minutes. Upper panel: impedance; lower panel: CFM record.

drop in systemic pressure which occurs frequently at the onset of perfusion. Three of these four patients were known to have arterial disease preoperatively, either coronary or cerebral.

A similar record, showing marked depression at the onset of perfusion with no other remarkable features, was obtained in a 6-year-old child who was irritable and confused following operation. A short episode of cardiac arrest occurred within the first $\mathbf{1 2}$ hours and it was apparent subsequently that the child had sustained gross neurological damage; it was impossible to determine the relative importance of the various neurological insults.

A further two patients (ages 50 and 64 years) had signs of a hemiplegia and a monoplegia respectively in the postoperative period. In both these patients the onset of perfusion produced little change in the CFM record, but a marked depression of activity occurred when the aortic clamp was removed at a time when the heart was not ejecting. Figure 6 is characteristic of these two records.

An elderly diabetic (aged 66 years) suffered an epileptic fit on the second postoperative day. Prior to this her postoperative course had been satisfactory with no grossly detectable neurological abnormality. The possibility of a transitory cardiac rhythm change preceding the convulsion could not be excluded. The record of this patient's cerebral function during surgery revealed a biphasic pattern at the onset of perfusion but the rest of the record was uneventful (Fig. 7).

A 1-year-old child suffered from convulsions and a left hemiparesis following circulatory arrest for $14 \frac{1}{2}$ minutes at a nasopharyngeal temperature of $27^{\circ} \mathrm{C}$. The only noticeable feature on the CFM record was a sudden change at the start of the arrest period, from an apparently normal trace to very low voltage activity which tended to diminish even further until the circulation was restarted, when cerebral activity recovered fairly rapidly (Fig. 8).

A mid-brain lesion was apparent postoperatively in a 36-year-old woman. This tracing (Fig. 9) was recorded at an unusually slow speed $(1.5 \mathrm{~mm} / \mathrm{min})$ and lacks detail, but apart from the elevation at the onset of perfusion, which persisted throughout the procedure, there is no major change. Studies of the oxygen content of blood drawn from the jugular bulb suggested that the lesion in this patient occurred at the onset of perfusion; details of this case will be included in a later report.

Three patients were confused for several days following operation. In a 63-year-old woman the CFM record throughout perfusion was unremarkable. A 56-year-old man required prolonged perfusion (203 minutes) and the mean level of the tracing fell slowly throughout this period. In the post-perfusion period an unexpected episode of ventricular fibrillation resulted in a short $(2 \mathrm{~min})$ 


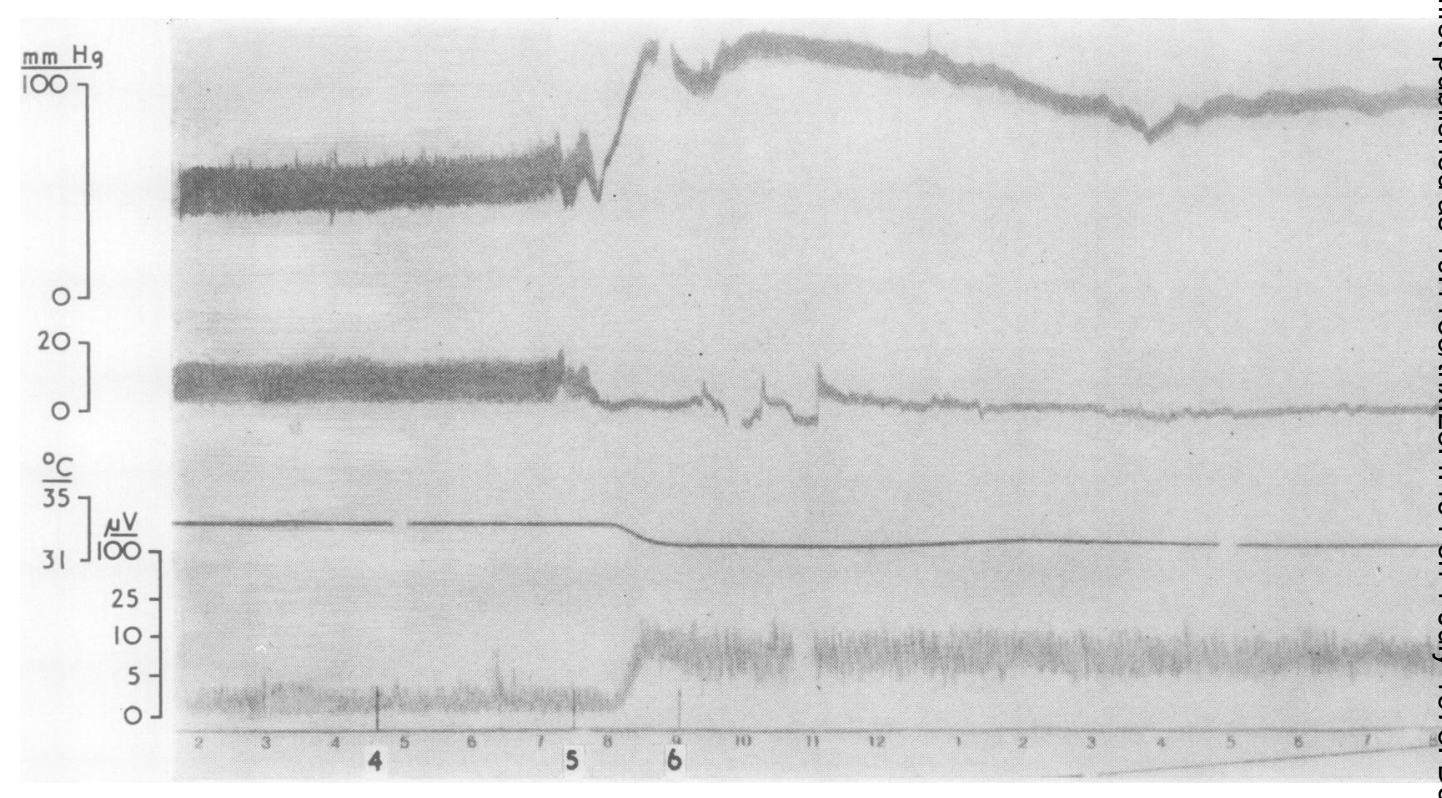

FIG. 10. Elevation of CFM record at the onset of perfusion (event marker No. 5) associated with an increase in arteri备 pressure in a 74-year-old man with severe heart failure. Records from above down: arterial blood pressure; superi@i caval pressure; nasopharyngeal temperature; CFM record.

record of virtually absent cerebral activity (Fig. 1b) before defibrillation restored an adequate circulation and the CFM tracing returned to a normal level. A 74-year-old man was hypotensive and in severe heart failure in the pre-perfusion period and the CFM tracing was low in mean level and amplitude. Following the onset of perfusion, the CFM record increased in both mean voltage and amplitude and remained unremarkable throughout the rest of the procedure (Fig. 10).

\section{DISCUSSION}

Previous reports of conventional EEG monitoring during cardiopulmonary bypass have stressed the frequency with which changes are observed during cannulation of the superior vena cava and at the onset of perfusion (Theye, Patrick, and Kirklin, 1957; Davenport, Arfel, and Sanchez, 1959; Adelman and Jacobson, 1960; Paton, Pearcy, and Swan, 1960; Storm van Leeuwen, Mechelse, Kok, and Zierfuss, 1961). Prior et al. (1971), reporting the use of the CFM during cardiac surgery, commented on the frequency with which changes were detected at the onset of perfusion and they attributed these to alterations in the depth of anaesthesia. In the present series, changes in the
CFM record during cannulation of the superiơ vena cava were seen infrequently but there was a high incidence of alteration at the onset of perfusion.

A number of factors could affect cerebra function at this time, including changes in the concentration of intravenous or inhalation anaesthetic agents, alterations in cerebral haem dynamics, arterial carbon dioxide tension, ten perature or haematocrit as well as unidentified factors related to perfusion. A subsequent report will describe which of the patterns illustrated the present paper can be attributed to one of other of the factors enumerated above.

In nine of the 10 cases with physical signs of neurological damage, an unusual degree of depre? sion of the CFM record occurred during perfi $\alpha^{0}$ sion; in six patients, this occurred at the onset perfusion and the depression was more prolong and profound than that recorded in patients witse out neurological damage. In one of these six cases (Fig. 7), the change was slight and no greatep than that seen in many patients whose postoperd tive course was entirely normal, and it was uncertain whether the cerebral lesion in this cas was sustained during surgery or postoperatively In the tenth case (Fig. 9) the cerebral lesion was 


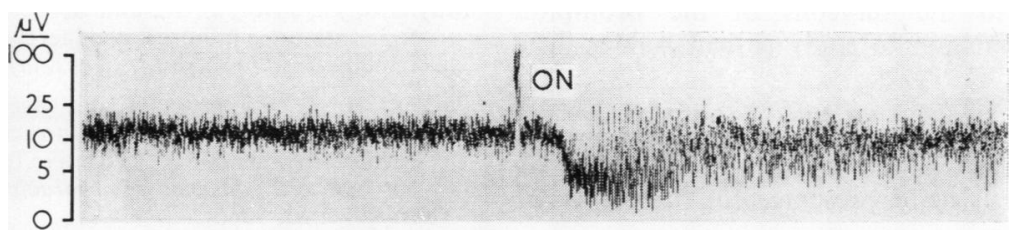

FIG. 11. Depression of CFM record at onset of perfusion associated with slow, high-voltage activity. This patient had no grossly detectable neurological defect in the postoperative period.

at mid-brain level and it is widely recognized that EEG abnormalities are not caused necessarily by mid-brain disorders.

It seems therefore that the CFM has provided an accurate record of the timing of cortical disturbances during perfusion. It is more difficult to relate the degree of detectable neurological abnormality to the duration or magnitude of the changes recorded on the CFM. The tracing in Fig. 11 is somewhat similar to that reproduced in Fig. 5, but whereas the patient whose record is shown in Fig. 11 had no grossly detectable neurological damage, Fig. 5 was recorded from a patient who was hemiplegic for several days postoperatively before making an apparently complete recovery. Depression of cortical activity probably represents a considerable disturbance but clinically obvious sequelae do not result inevitably (compare cerebral concussion).

The onset of extracorporeal circulation is accompanied by many changes which could exert an adverse influence on cerebral function. An obvious possibility is a sudden drop in arterial blood pressure with a change from pulsatile to non-pulsatile perfusion. This fall in pressure is common in spite of high flow rates from the perfusion apparatus $(80 \mathrm{ml} / \mathrm{kg} / \mathrm{min}$ or more $)$. Although the cerebral circulation has the capacity to autoregulate so that cerebral blood flow is maintained in the face of wide changes in arterial pressure (Lassen, 1959), there is a limit below which cerebral blood flow falls. The adjustments cannot compensate for very rapid changes in pressure (Schneider, 1963) and the degree to which the cerebral vessels can dilate is probably reduced in the elderly, especially in those with cerebrovascular disease (Bromage, 1953). Acute hypotension was documented in four of the five patients in whom major changes in cerebral activity occurred at the onset of perfusion; four of these patients were aged more than 50 years and three had a history of arterial disease.

An alternative cause for neurological damage at the onset of cardiopulmonary bypass could be emboli from the perfusion apparatus. Since January 1971, additional filters have been added to the perfusion system and the incidence of depression of the CFM record at the onset of perfusion has changed from 7/33 (21.2\%) in 1969 and 1970 to $13 / 107(12 \cdot 2 \%)$ in 1971 and 1972 . There has also been a very obvious reduction in the incidence of important neurological injury in this unit since early 1971, although precise figures are not available yet. It seems difficult to deny the conclusion that emboli from the perfusion system may have been responsible in part for neurological damage, even though acute hypotension could have been an additional contributory factor.

In three patients, the neurological disturbance appeared to occur during perfusion. In one case, circulatory arrest without adequate protection from hypothermia was probably responsible, whereas in the other two instances, release of the aortic clamp was associated with depression of cerebral activity. Emboli of air, clot or calcium trapped in the left heart or root of the aorta could have been responsible; alternatively, acute hypotension may have occurred as the aortic valve was 'tripped' in one case, and the perfusion pressure fell abruptly.

A notable feature has been the complete absence of obvious changes detected during the re-establishment of a spontaneous circulation, when the left ventricle is beginning to eject. Manoeuvres to remove air from the left heart are notoriously ineffective (Padula, Eisenstat, Bronstein, and Camishion, 1971; Lawrence, McKay, and Sherensky, 1971) and in several of the patients in this series, air was seen frothing out of the puncture wound in the root of the aorta with every ventricular contraction. Although air trapped in the circulation can cause extensive neurological damage, the lack of change in the CFM record with the onset of left ventricular ejection suggests that cerebral air embolism is rarely of serious magnitude in this unit. 
I am grateful to the surgeons of the Brompton Hospital for permission to study patients under their care.

The work has been supported by a grant from the Medical Research Council and the results will be included in a thesis to be presented for the degree of M.D. (Cantab.).

\section{REFERENCES}

Adelman, M. H., and Jacobson, E. (1960). Electroencephalography in cardiac surgery. American Journal of Cardio$\log y, 6,763$.

Branthwaite, M. A. (1972). Neurological damage related to open-heart surgery: a clinical survey. Thorax, 27, 748.

Brazier, M. A. B. (1961). The EEG in open-heart surgery and in surgery for aortic and cerebral aneurysms. In Cerebral Anoxia and the Electroencephalogram, p. 256, edited by $\mathrm{H}$. Gastaut, and J. S. Meyer. Thomas, Springfield, Illinois.

Bromage, P. R. (1953). Some electroencephalographic changes associated with induced vascular hypotension. Proceedings of the Royal Society of Medicine, 46, 919.

Davenport, H. T., Arfel, G., and Sanchez, F. R. (1959). The electroencephalogram in patients undergoing open heart surgery with heart/lung bypass. Anesthesiology, 20, 674.

Fischer-Williams, M., and Cooper, R. A. (1964). Some aspects of electroencephalographic changes during open-heart surgery. Neurology (Minneapolis), 14, 472.

Lassen, N. A. (1959). Cerebral blood flow and oxygen consumption in man. Physiological Review, 39, 183.

Lawrence, G. H., McKay, H. A., and Sherensky, R. T. (1971). Effective measures in the prevention of intraoperative aeroembolus. Journal of Thoracic and Cardiovascular Surgery, 62, 731.
Maynard, D., Prior, P. F., and Scott, D. F. (1969). Devicew for continuous monitoring of cerebral activity in resusci= tated patients. British Medical Journal, 4, 545.

Padula, R. T., Eisenstat, T. E., Bronstein, M. H., and Camishion, R. C. (1971). Intracardiac air following cardiotomy. Location, causative factors, and a method for removal. Journal of Thoracic and Cardiovasculan Surgery, 62, 736.

Pampiglione, G. and Waterston, D. J. (1961). EEG observa $\overrightarrow{0}$ tions during changes in venous and arterial pressure.In Cerebral Anoxia and the Electroencephalogram, p. 250 $\vec{\omega}$ edited by $\mathrm{H}$. Gastaut and J. S. Meyer. Thomas? Springfield, Illinois.

Paton, B., Pearcy, W. C., and Swan, H. (1960). The importi ance of the electroencephalogram during open cardiaco surgery with particular reference to superior vena caval obstruction. Surgery, Gynecology and Obstetrics, 111, $197 \stackrel{\circ}{\circ}$

Prior, P. F., Maynard, D. E., Sheaff, P. C., Simpson, B. R. Strunin, L., Weaver, E. J. M., and Scott, D. F. (1971) Monitoring cerebral function: clinical experience withnew device for continuous recording of electrica activity of brain. British Medical Journal, 2, 736.

Sadove, M. S., Becka, D., and Gibbs, F. A. (1967). Electro $\vec{c}$ encephalography for Anaesthesiologists and Surgeons. Pitman, London.

Schneider, M. (1963). Critical blood pressure in the cerebra circulation. In Selective Vulnerability of the Brain in Hypoxaemia (A Symposium organized by the Councie for International Organizations of Medical Sciences edited by J. P. Schade and W. H. McMenemey pp. 7-20을 Blackwell, Oxford.

Storm van Leeuwen, W., Mechelse, K., Kok, L., and Zierfuss, E. (1961). EEG during heart operations with artificial circulation. In Cerebral Anoxia and the Electro encephalogram, p. 268, edited by H. Gastaut and J. S Meyer. Thomas, Springfield, Illinois.

Theye, R. A., Patrick, R. T., and Kirklin, J. W. (1957) The electroencephalogram in patients undergoing open intracardiac operations with the aid of extracorporeap circulation. Journal of Thoracic Surgery, 34, 709. 